\title{
Anisotropic Volume Rendering Using Intensity Interpolation
}

\author{
Tae-Young Kim ${ }^{1}$, Byeong-Seok Shin², Yeong Gil Shin ${ }^{1}$ \\ ${ }^{1}$ School of Computer Science and Engineering, Seoul National University \\ San 56-1, Shilim-Dong, Kwanak-Gu, Seoul 151-742, Korea \\ \{tykim,yshin\}@cglab.snu.ac.kr \\ ${ }^{2}$ Dept. of Computer Science and Engineering, Inha University \\ 253 YongHyeon-Dong Nam-Gu, Inchon, Korea \\ bsshin@inha.ac.kr
}

\section{Introduction}

In medical applications, anisotropic volume data sets are commonly used, of which the resolution in an axial plane is higher than that in the direction perpendicular to it. In this paper, we propose an efficient anisotropic volume rendering method based on intensity interpolation that obtains the intensity value at an interpolated position lying between voxels in two consecutive slices from the intensity values of the voxels. Unlike density interpolation method, it does not require long preprocessing time and extra memory for storing the interpolated slices. Experimental results show that this method improves rendering speed without significantly sacrificing image quality.

\section{Anisotropic Volume Rendering Using Intensity Interpolation}

In order to manipulate the anisotropic volume in rendering time, intensity calculation on missing voxels is required. Fig. 1 compares our method with a conventional density interpolation method to compute the intensity of an interpolated sample $v_{i}$ divided internally as $m:(1-m)$ between the voxels in two adjacent slices, $v_{k}$ and $v_{k+1}$. In the figure, $d\left(v_{i}\right), N\left(v_{i}\right)$ and $I\left(v_{i}\right)$ is a density value, a normal vector and an intensity value of $v_{i}$, respectively. Since the conventional method computes $I\left(v_{i}\right)$ from

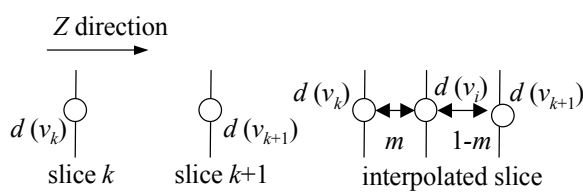

(a) Initial voxels

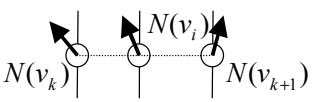

(c) Normal vector calculation (b) Density value interpolation

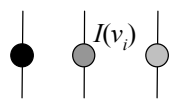

(d) Intensity value calculation

Conventional method

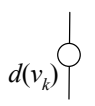

(a) Initial voxels

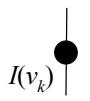

(c) Intensity value calculation

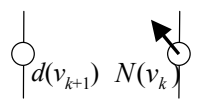

(b) Normal vector calculation
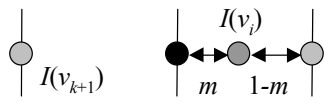

(d) Intensity value interpolation

\section{Intensity interpolation}

Fig. 1. Comparison of intensity calculation process. 
$N\left(v_{i}\right)$ obtained by interpolating density values of $v_{i}$ and its neighbors, the normal calculation, with additional density interpolation is required for each interpolation position. On the other hand, intensity interpolation method computes $I\left(v_{i}\right)$ from $I\left(v_{k}\right)$ and $I\left(v_{k+1}\right)$. It is not necessary to compute density values and normal vectors for intermittent samples. The normal calculation is only performed on the original voxels.

Consider the equation $I\left(v_{k}\right)=K_{a} I_{a}+K_{d}\left(L \bullet N\left(v_{k}\right)\right)$ where $K_{a}$ is an ambientreflection coefficient, $I_{a}$ is an ambient-light intensity, $K_{d}$ is a diffuse-reflection coefficient, $L$ is a light vector. Equation (1) proves that the intensity value $\widetilde{I}\left(v_{i}\right)$, computed by intensity interpolation is equivalent to the intensity value calculated from the normal vector $\tilde{N}\left(v_{i}\right)$ :

$$
\begin{aligned}
\widetilde{I}\left(v_{i}\right) & =I\left(v_{k}\right)(1-m)+I\left(v_{k+1}\right) m \\
& =\left(K_{a} I_{a}+K_{d}\left(L \bullet N\left(v_{k}\right)\right)\right)(1-m)+\left(K_{a} I_{a}+K_{d}\left(L \bullet N\left(v_{k+1}\right)\right)\right) m \\
& =K_{a} I_{a}(1-m)+K_{a} I_{a} m+K_{d} L \bullet\left(N\left(v_{k}\right)(1-m)+N\left(v_{k+1}\right) m\right) \\
& =K_{a} I_{a}+K_{d} L \bullet\left(N\left(v_{k}\right)(1-m)+N\left(v_{k+1}\right) m\right) \\
& =K_{a} I_{a}+K_{d}\left(L \bullet \tilde{N}\left(v_{i}\right)\right)
\end{aligned}
$$

Compare the normal vectors along $Z$-axis of these methods. Following equations represent the normal vectors $N_{Z}\left(v_{i}\right)$ and $\tilde{N}_{Z}\left(v_{i}\right)$ (by equation (1)), which are for the conventional method and the intensity interpolation method, respectively.

$$
\begin{aligned}
N_{Z}\left(v_{i}\right) & =\frac{2\left(d\left(v_{k+1}\right)-d\left(v_{k}\right)\right)}{\lambda} \\
\tilde{N}_{Z}\left(v_{i}\right) & =N_{Z}\left(v_{k}\right)(1-m)+N_{Z}\left(v_{k+1}\right) m \\
& =\frac{\left(d\left(v_{k+1}\right)-d\left(v_{k-1}\right)\right)(1-m)+\left(d\left(v_{k+2}\right)-d\left(v_{k}\right)\right) m}{2 \lambda}
\end{aligned}
$$

In the equations, more samples are referenced for calculating $\widetilde{N}_{Z}\left(v_{i}\right)$ compared to $N_{Z}\left(v_{i}\right)$. Thus the smoother images are obtained by the intensity interpolation.

\section{Experimental Results}

To evaluate the performance of our method, we compare it with other approaches [1][3] such as interpolating density values in the preprocessing step (P-Interp), interpolating them in the rendering time (R-Interp) and transforming the anisotropic volume to isotropic one by scaling matrix (S-Matrix). These are implemented on an $800 \mathrm{MHz}$ Pentium III PC with 512 MB of main memory. As a rendering algorithm, the scanlinebased shear-warp factorization [1] is used.

Table 1 compares processing time and storage. Among these methods, only PInterp requires preprocessing time for creating missing slices, and it increases rendering time due to the memory access overhead. R-Interp takes longer time than our method due to the conventional interpolation process. S-Matrix is the fastest because of the long sampling distance. Table 2 shows that our method is much more efficient than R-Interp in the images requiring a lot of samples.

Fig. 2 shows the image quality. Compared to P-Interp and R-Interp, our method alleviates staircases while preserving detail information. On the other hand, S-Matrix produces excessive staircase artifacts, thus it is not practical in medical applications. 
Table 1. Comparison of time and storage.

\begin{tabular}{clcccc}
\hline Data & \multicolumn{1}{c}{ Performance Test } & Ours & P-Interp & R-Interp & S-Matrix \\
\hline \multirow{3}{*}{ Airway } & Pre-interpolation Time (ms) & - & 12684 & - & - \\
& Average Rendering Time (ms) & 1108 & 1486 & 1513 & 673 \\
& Storage (MB) & 71.8 & 240.8 & 71.8 & 71.8 \\
\hline
\end{tabular}

Table 2. Comparison of average rendering times between our method and R-Interp according to the number of contributed samples (colon data with $512 \times 512 \times 141$ resolution).

\begin{tabular}{ccccc}
\hline Structure & Samples & Ours $(\mathrm{ms})$ & R-Interp (ms) & Speedup \\
\hline Colon & $0.6 \mathrm{M}$ & 1362 & 1992 & 1.46 \\
Skin and Bone & $8.1 \mathrm{M}$ & 5235 & 8802 & 1.68 \\
Semitransparent Skin & $13.3 \mathrm{M}$ & 8230 & 15047 & 1.83 \\
\hline
\end{tabular}

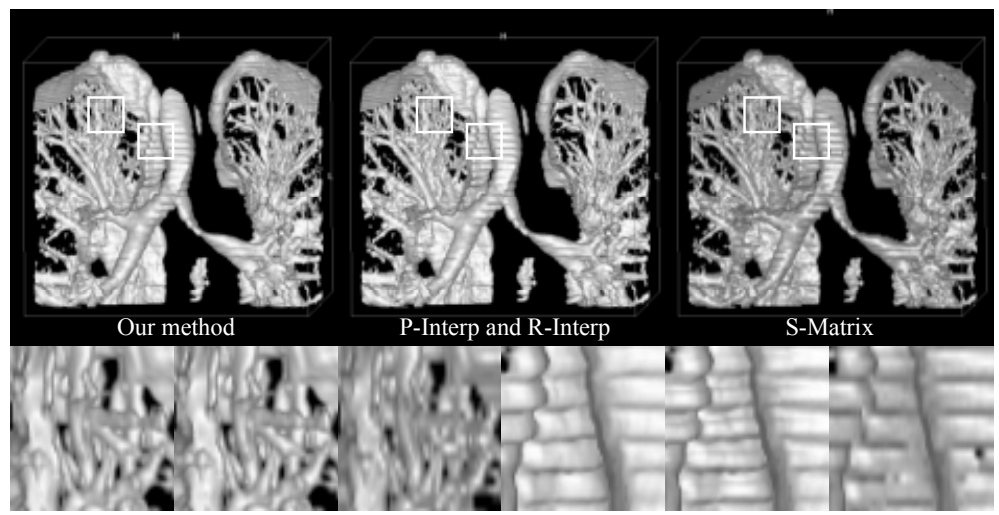

Fig. 2. Comparison of image quality on detailed parts (airway data): (left) our method; (middle) P-Interp and R-Interp; (right) S-Matrix.

\section{Conclusion}

We have presented an efficient way of calculating the intensity value on each sample in visualizing anisotropic volume data sets. We are currently extending our approach to avoid overblurring on high frequency area.

\section{References}

1. Lacroute P., Levoy, M.: Fast Volume Rendering Using a Shear-Warp Factorization of the Viewing Transformation. Computer Graphics (SIGGRAPH '94 Proceedings), Orlando, Florida, July (1994) 451-458

2. Mueller K., Shareef N., Huang J., Crawfis R.: High-quality splatting on rectilinear grids with efficient culling of occluded voxels. IEEE Transactions on Visualization and Computer Graphics, 5(2) (1999) 116-134

3. Pfister, H., Hardenbergh, J., Knittel, J., Lauer, H., Seiler, L.: The VolumePro Real-Time RayCasting System. Computer Graphics (SIGGRAPH '99 Proceedings), Los Angeles, CA, August (1999) 251-260 